\title{
Smart Button: A Wearable System for Assessing Mobility in Elderly
}

\author{
Armen Dzhagaryan, Aleksandar Milenkovic, \\ Emil Jovanov \\ Electrical and Computer Engineering Department \\ The University of Alabama in Huntsville \\ Huntsville, AL
}

\author{
Mladen Milosevic \\ Philips Research North America \\ Briarcliff Manor, NY
}

\begin{abstract}
Continuous advances in sensors, semiconductors, wireless networks, mobile and cloud computing enable the development of integrated wearable computing systems for continuous health monitoring. These systems can be used as a part of diagnostic procedures, in the optimal maintenance of chronic conditions, in the monitoring of adherence to treatment guidelines, and for supervised recovery. In this paper, we describe a wearable system called Smart Button designed to assess mobility of elderly. The Smart Button is easily mounted on the chest of an individual and currently quantifies the Timed-Upand-Go and 30-Second Chair Stand tests. These two tests are routinely used to assess mobility, balance, strength of the lower extremities, and fall risk of elderly and people with Parkinson's disease. The paper describes the design of the Smart Button, parameters used to quantify the tests, signal processing used to extract the parameters, and integration of the Smart Button into a broader mHealth system.
\end{abstract}

Keywords-mobile sensing; health monitoring; wearable devices; timed-up-and-go test; 30-second chair stand test.

\section{INTRODUCTION}

Time-Up-and-Go (TUG) and 30-second Chair Stand (30SCS) tests are simple, easy to administer, and frequently used tests for assessing balance, mobility, and functional lower extremity strength in elderly, geriatric patients, and people with Parkinson's disease [1]-[3]. The TUG test measures the time a person takes to perform the following tasks: rise from a chair, walk three meters, turn around, walk back to the chair, and sit down. Longer TUG times have been associated with mobility impairments and increased fall risks [1], [4], [5]. Adults without balance problems can perform this test in less than 10 seconds. Alternatively, adults with mobility difficulty may require more than 30 seconds. The TUG times are also sensitive to therapeutic interventions, e.g., in Parkinson's patients [6], [7]. Whereas the test has been proven valuable in early assessment of balance and mobility, it is limited as its only outcome is the time to complete the test.

The 30SCS test, based on the five-times-sit-to-stand test [3], measures the number of stands a person can perform during a 30 second interval. The primary goal of this test is to measure the lower extremity strength, but it can also be

This work was supported in part by the U.S. National Science Foundation under grants CNS-1205439 and CNS-1217470. sensitive to speed, balance, and mobility [8]-[10]. The test is limited as its only quantitative outcome is the number of stands completed within 30 seconds.

Instrumented Timed-Up-and-Go (iTUG) [11], [12] and Chair Stand tests [13], [14] have been recently introduced. In both tests dedicated devices are used to capture signals from inertial sensors that are later analyzed by health care professionals. A number of additional parameters can be derived that can better indicate gait and balance impairments, including Sit-to-Stand (S2ST) duration, Stand-to-Sit (ST2S) duration, and the amplitude range of anterior-posterior, to name just a few. The iTUG has proven to be sensitive to pathologies [11], [12] and useful in fall risk prediction [15]. In our prior work [16] we introduced a smartphone application called sTUG (smartphone-enabled Timed-Up-and-Go). With sTUG, a smartphone is mounted on the subject's chest and the application monitors and processes signals from the smartphone's inertial sensors to extract key parameters that quantify the TUG test. Though this application has been well received by health care professionals and deployed in several institutions, smartphones are relatively bulky and require mounting using a chest strap which could be a problem for elderly people and women.

In this paper, we introduce a small wearable computing system called Smart Button. The Smart Button consists of a 9 degrees of freedom (DOF) inertial sensor with a 3-axis accelerometer, a 3-axis gyroscope, and a 3-axis magnetometer; a system-on-a-chip that integrates a processor, memory, and I/O peripherals; a Bluetooth interface; and a battery with a charger. Due to its small form factor and weight, the Smart Button can be easily mounted on a subject's chest in the form of a button. The Smart Button's firmware interfaces the inertial sensors, processes the signals from the sensors, and extracts key parameters that quantify the TUG and 30SCS tests. The Smart Button also communicates to a personal device via a Bluetooth interface. The personal device runs an application that controls the Smart Button by (a) selecting a test to be conducted, (b) starting the test, and (c) retrieving the parameters once the test has been completed. The personal device application displays the test results and logs them locally on the device. Optionally, the test results can be automatically uploaded to a medical server for long term storage and analysis. 
To quantify the TUG test, the Smart Button analyzes the signals from the inertial sensors and extracts the following parameters: (a) the total duration of the TUG test, (b) the total duration of sit-to-stand and stand-to-sit transitions, (d) maximum angular velocities and maximum angles during the transitions, and (e) the number of steps made by the subject during the test. For the 30SCS test, the Smart Button determines the number of chair stands in 30 seconds. In addition, it quantifies each chair stand by determining the duration of transitions, maximum angular velocities, and maximum angles.

The Smart Button could be of great interest to elderly and individuals with Parkinson's disease, as well as to health care professionals. The Smart Button is an inexpensive solution for quantifying mobility of patients, requires minimum setup (pairing with a personal device), provides an instantaneous feedback to the user in a form of a report, and supports automatic logging on servers, if desired. The test can be conducted anytime by professionals or individual users. The Smart Button's firmware can be easily upgraded to support a number of different tests and procedures. With long term storage and analysis of the tests on the medical servers, patients, informal caregivers, and health care professionals can gain insights into overall wellness of the subjects, evolution of disease, or evaluate the impact of therapeutic interventions.

The rest of this paper is organized as follows. Section II describes the TUG and 30SCS tests. Section III describes the system architecture including the Smart Button, personal device, and the mHealth server. Section IV discusses body instrumentation, defines the parameters for quantifying the tests, and describes how the Smart Button extracts them. Section V describes testing of the Smart Button and shares some results, and Section VI concludes the paper.

\section{Mobility Tests}

\section{A. Timed-Up-and-Go Test}

The Timed-Up-and-Go (TUG) test is routinely used to assess mobility in elderly and Parkinson's patients. Fig. 1 illustrates typical phases of a TUG test. Patients wear their regular footwear and can use a walking aid if needed. The test begins by having the patient sit back in a standard armchair with a clearly marked line 3 meters away on the floor. On a command "Go" the patient should stand up from the chair, walk to the line on the floor at his/her normal pace, turn and walk back to the chair, and sit down. A health care professional who administers the test uses a stopwatch to measure time to complete the test, from the command "Go" to the moment the patient is sitting back in the chair. The test outcome is the time to complete the test. An older adult who takes more than 12 seconds to complete the TUG test is at high risk for falling [17]. In addition, the health care professionals typically observe the patient's postural stability, gait, stride length, and sway to fill in a form identifying slow, tentative pace, loss of balance, short strides, little or no arm swing, shuffling, en bloc turning, or improper use of walking aids.

\section{B. 30-Second Sit-Stand Test}

The 30-Second Chair Stand (30SCS) test is used to assess leg strength and endurance. Fig. 2 illustrates a typical single cycle in the 30SCS test. The test is conducted using a straight back chair without arm rests and a stopwatch. The patient is sitting in the middle of the chair with feet flat on the floor with hands placed on the opposite shoulders and crossed at the wrists. On the command "Go" the patient rises to a full standing position and then sits back down holding arms against his/her chest. The patient keeps repeating these steps for 30 seconds. The outcome of the test is the number of times the patient comes to a full standing position in 30 seconds. Table 1 gives below average scores as a function of gender and age used to determine whether the patient is at high risk for falls [18].

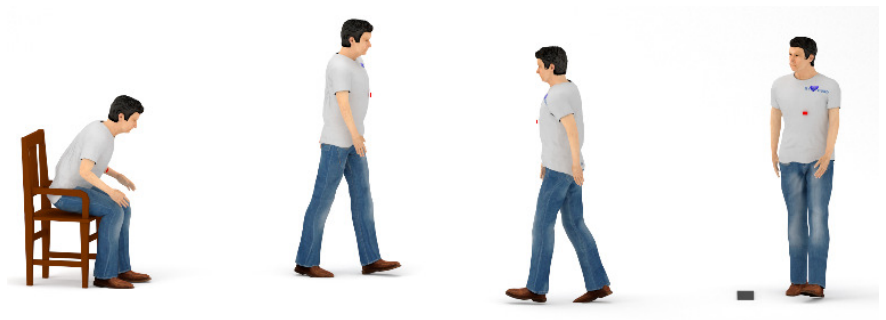

Fig. 1. Timed-Up-and-Go test phases.

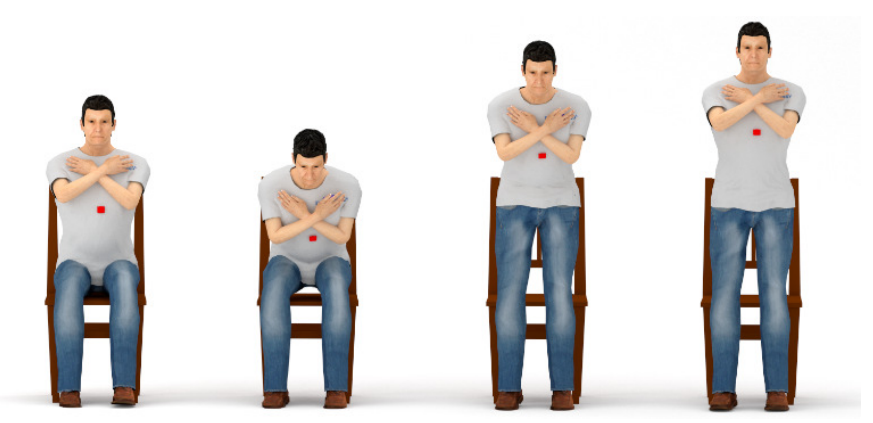

Fig. 2. 30 Second Sit Stand phases.

TABLE I. 30 SeCond Chair Stand: Below Average Scores

\begin{tabular}{|c|l|l|}
\hline Age & Men & Women \\
\hline $60-64$ & $<14$ & $<12$ \\
\hline $64-69$ & $<12$ & $<11$ \\
\hline $70-74$ & $<12$ & $<10$ \\
\hline $75-79$ & $<11$ & $<10$ \\
\hline $80-84$ & $<10$ & $<9$ \\
\hline $85-89$ & $<8$ & $<8$ \\
\hline $90-94$ & $<7$ & $<4$ \\
\hline
\end{tabular}

\section{SYSTEM ARCHITECTURE}

The proposed system for automated assessment of mobility utilizes a 3-tiered architecture described in Fig. 3. At Tier 1 we use a Smart Button - a wearable Bluetooth-enabled embedded computer with inertial sensors. The Smart Button is paired with a personal device at Tier 2. The personal device can be a smartphone, a tablet, or a personal computer. In this paper, we use a smartphone as the personal device. The personal device connects via the Internet to an mHealth server at Tier 3. 
The Smart Button is designed with a goal to provide an automated assessment of mobility by supporting multiple tests. It incorporates the following components: (a) a Teensy 3.1 board featuring a MK20DX256VLH7 SoC that includes a 32bit ARM Cortex-M4 processor running at $72 \mathrm{MHz}, 256 \mathrm{~KB}$ of flash memory, and $64 \mathrm{~KB}$ of RAM memory; (b) a Bluetooth radio; (c) a 9-DOF inertial sensor including a 3D accelerometer, a 3D magnetometer, and a 3D gyroscope; and (d) a Li-Ion battery with a charger. The form factor of our current Smart Button prototype is $50.8 \times 28.6$ x $15.9 \mathrm{~mm}$ and the weight is $40 \mathrm{~g}$. However, we anticipate that custom built module will fit the form factor of a regular-sized button.

The Smart Button's firmware includes modules for handling Bluetooth communication, sensor interfacing, and onboard signal processing for parameters extraction in order to quantify the supported tests. Once the Smart Button is powered up, it waits for commands from the personal device over the Bluetooth interface. These commands indicate the beginning of the TUG test or the 30SCS test. In a normal operating mode signals from the inertial sensors are sampled with a frequency of $100 \mathrm{~Hz}$ and read through an $\mathrm{I}^{2} \mathrm{C}$ interface. From these signals the Smart Button also derives the orientation using the algorithm described in [19]. The Smart Button pre-processes the signals from the inertial sensors using an average filter with a sliding window of 21 samples, detects characteristic events of interest (beginning and end of transitions, steps, and others), time stamps these events, and derives key parameters of interest for each test. The Smart Button autonomously detects the completion of the tests and then sends the processed parameters to the personal device. In addition to the normal operating mode, the Smart Button can operate in a debug mode in which raw signals from the inertial sensors and derived signals are streamed to the personal device.

The smartphone runs our modified sTUG application [16]. The application interfaces the Smart Button over the Bluetooth as well as the mHealth server over its broadband or WLAN interfaces. In a clinical setting, a health care professional starts the application, selects a patient to undergo a test, selects the test to be performed (TUG or 30SCS), and then starts the test by saying "Go" and pressing a Start button in the application at the same time. The application sends an appropriate command to the Smart Button and waits for the test results to come back. Once the results are received, they are immediately displayed on the smartphone (Fig. 3), as well as logged into a text file.

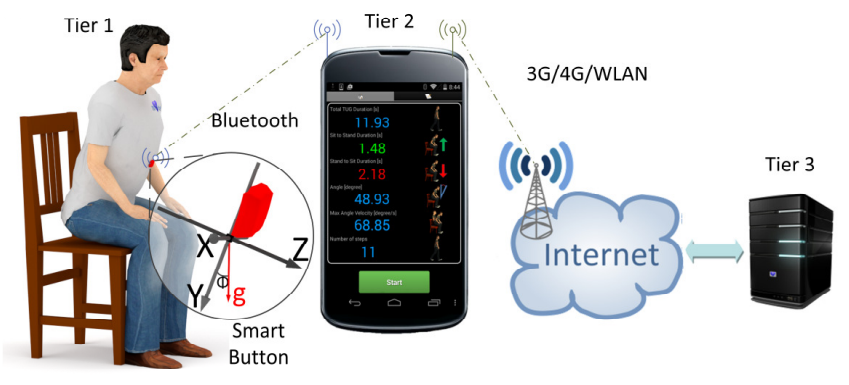

Fig. 3. Smart Button -based system for mobility assessment.

A test descriptor that includes the time and date when the test is taken as well as all the parameters is uploaded into a database on the mHealth server. The mHealth server is a collection of virtual machines providing various services including long-term data storage, database management, authentication, secure communication, as well as data retrieval and visualization [20].

\section{SMART BUTTON: From SigNALS TO INFORMATION}

\section{A. Body instrumentation}

We instrument a subject by placing the Smart Button on his or her chest. To remain fixed to the trunk during a test, the button is placed on an elastic chest strap. Alternatively, it can be attached to the clothes using a Velcro strap or a clip, providing clothes are not loose. Additional placements are possible, such as the subject's back. In this paper, we assume that the Smart Button is placed close to sternum in an upright position, attached to the clothes using a clip.

Fig. 3 shows orientation of the inertial sensors on the Smart Button relative to the subject. The $z$ axis corresponds to the sagittal axis, the $y$ axis corresponds to the longitudinal axis, and the $x$ axis corresponds to the frontal axis of the human body. The Smart Button's inertial 9-DOF device includes an accelerometer, a gyroscope, and a magnetic sensor. The accelerometer sensor measures $x, y$, and $z$ components of the acceleration of the subject's upper trunk and we use these signals to determine the subject's upper body position. Additionally, changes in accelerometer magnitudes are used to detect and eliminate false movements, such as swinging the body without actually getting up. The gyroscope measures angular movements, specifically the rotation around three axes: roll ( $x$ axis), yaw ( $y$ axis), and pitch ( $z$ axis). In our setup, we record angular speeds from the gyroscope and use these signals to detect and locate important phases during postural transitions in the TUG and 30SCS tests. The magnetic sensor is sensing the Earth's magnetic field. In our setup we use the signal to detect changes in orientation and turning around in the TUG test.

\section{B. TUG and 30SCS Characterization}

To characterize the TUG, we start from a set of parameters suggested by Weiss et. al [11] and its extension proposed in the sTUG application [16]. In addition to these, we add several new parameters, including the number of steps made during walking. We also distinguish the duration of the test, $d . T U G$, measured from the moment the command "Go" is issued until the subject is back in the sitting position, and the total active time, d.aTUG, measured from the moment the subject starts leaning forward until the subject is back in the sitting position. The difference between the two gives the time the subject needs to react to a command.

Table 2 gives a full set of parameters we extract to characterize the TUG test. To characterize posture transitions we measure the duration of the sit-to-stand transition (d.S2ST) and the duration of the stand-to-sit transition (d.ST2S). The sitto-stand transition is further characterized by the duration of a leaning forward phase (d.LF) and the duration of a lifting up phase (d.LT), the maximum upper trunk angle (a.S2ST) at the end of the lean forward phase, the maximum angular velocities in the lean forward (v.LF) and the lift up (v.LT) phases. Similarly, we determine the duration of separate phases of the 
stand-to-sit transition, a preparing to sit phase (d.PS) and a sitting down phase (d.SD).

TABLE II. PARAMETERS FOR TUG CHARACTERIZATION

\begin{tabular}{|c|c|c|}
\hline $\begin{array}{l}\text { Parame } \\
\text {-ter }\end{array}$ & Description & Units \\
\hline d.TUG & $\begin{array}{l}\text { Total duration of the TUG test } \\
\text { (from "Go" to the completion of the test) }\end{array}$ & seconds \\
\hline d.aTUG & $\begin{array}{l}\text { Total duration of the active TUG (from the } \\
\text { moment the subject starts leaning forward } \\
\text { to the completion of the test) }\end{array}$ & seconds \\
\hline d.S2ST & $\begin{array}{l}\text { Total duration of the sit-to-stand transition; } \\
\text { d.S2ST }=\text { d.LF }+ \text { d.LT }\end{array}$ & seconds \\
\hline d.LF & $\begin{array}{l}\text { Duration of the lean forward phase in the } \\
\text { sit-to-stand transition (terminated when the } \\
\text { upper trunk angular velocity reaches a zero) }\end{array}$ & seconds \\
\hline d.LT & $\begin{array}{l}\text { Duration of the lift up phase } \\
\text { in the sit-to-stand transition }\end{array}$ & seconds \\
\hline a.S2ST & $\begin{array}{l}\text { Maximum change of the trunk angle } \\
\text { in the lean forward phase }\end{array}$ & degrees \\
\hline v.LF & $\begin{array}{l}\text { Maximum angular velocity } \\
\text { during the lean forward phase }\end{array}$ & degrees/s \\
\hline v.LT & $\begin{array}{l}\text { Maximum angular velocity } \\
\text { during the lift up phase }\end{array}$ & degrees/s \\
\hline d.ST2S & $\begin{array}{l}\text { Duration of the stand-to-sit transition; } \\
\text { d.ST2S }=\text { d.PS }+ \text { d.SD }\end{array}$ & seconds \\
\hline d.PS & $\begin{array}{l}\text { Duration of the prepare-to-sit phase } \\
\text { in the stand-to-sit transition }\end{array}$ & seconds \\
\hline d.SD & $\begin{array}{l}\text { Duration of the sit-down phase } \\
\text { in the stand-to-sit transition }\end{array}$ & seconds \\
\hline n.STEP & Number of steps during walking phase & steps \\
\hline
\end{tabular}

TABLE III. PARAMETERS FOR 30SCS CHARACTERIZATION

\begin{tabular}{|c|c|c|}
\hline $\begin{array}{l}\text { Parame- } \\
\text { ter }\end{array}$ & Description & Units \\
\hline n.CST & Number of chair stands in 30 seconds & number \\
\hline d.S2STi & $\begin{array}{l}\text { Total duration of the } \mathrm{i}^{\text {th }} \text { sit-to-stand } \\
\text { transition: d.S2ST } \mathrm{ST}_{\mathrm{i}}=\text { d. } \mathrm{LF}_{\mathrm{i}}+\text { d. } \mathrm{LT}_{\mathrm{i}}\end{array}$ & seconds \\
\hline d. $L F_{i}$ & $\begin{array}{l}\text { Duration of the lean forward phase } \\
\text { in the sit-to-stand transition }\end{array}$ & seconds \\
\hline d. $\mathrm{LT}_{\mathrm{i}}$ & $\begin{array}{l}\text { Duration of the lift up phase } \\
\text { in the sit-to-stand transition }\end{array}$ & seconds \\
\hline a.S2STi & $\begin{array}{l}\text { Maximum change of the trunk angle } \\
\text { in the lean forward phase }\end{array}$ & degrees \\
\hline v.LF ${ }_{i}$ & $\begin{array}{l}\text { Maximum angular velocity } \\
\text { during the lean forward phase }\end{array}$ & degrees/s \\
\hline v.LT $\mathrm{LT}_{\mathrm{i}}$ & $\begin{array}{l}\text { Maximum angular velocity } \\
\text { during the lift up phase }\end{array}$ & degrees/s \\
\hline d. $\mathrm{ST}_{\mathrm{i}}$ & Total duration of standing phase & seconds \\
\hline d.ST2S & $\begin{array}{l}\text { Duration of the stand-to-sit transition; } \\
\text { d.ST2 } S_{i}=\text { d.PS } \\
\text { i }+ \text { d.SD }\end{array}$ & seconds \\
\hline d.PS & $\begin{array}{l}\text { Duration of the prepare-to-sit phase } \\
\text { in the stand-to-sit transition }\end{array}$ & seconds \\
\hline d. $\mathrm{SD}_{\mathrm{i}}$ & $\begin{array}{l}\text { Duration of the sit-down phase } \\
\text { in the stand-to-sit transition }\end{array}$ & seconds \\
\hline d. SIT $_{\mathrm{i}}$ & Total durationg of sitting phase & seconds \\
\hline
\end{tabular}

To characterize the 30SCS test we use a set of parameters shown in Table 3. The main outcome of the test is the number of sit-to-stand transitions made in 30 seconds. However, we can characterize each cycle $(i=1,2, \ldots \mathrm{N})$ that includes a sit-tostand transition followed by a stand-to-sit transition. In addition, the subject may take some time in either standing
(d.STi) or sitting state (d.SITi), before the next transition is undertaken. By characterizing each individual cycle, we can quantify the subject's ability to keep the pace repeating chair stands, an indicator of the subject's lower extremity strength.

\section{Parameter Extraction}

Fig. 4 shows raw acceleration, angular velocity, and orientation signals captured and computed by the Smart Button in the debug mode during an entire TUG test. When the command Go is issued, $\mathrm{T}_{\mathrm{GO}}$ timestamp is captured. Next, the Smart Button is looking for the beginning of the sit-to-stand transition $\left(\mathrm{T}_{\mathrm{bS} 2 \mathrm{ST}}\right)$ by searching for a change in the angular velocity around the frontal axis (the $x$ component of the gyroscope). This change is caused by leaning forward as the subject prepares to stand up. Additionally, acceleration thresholds are checked to ensure that the leaning forward signature is caused by an actual motion rather than by swinging forward while still in sitting position. The angular velocity during the sit-to-stand transition has a distinct profile as illustrated in Fig. 5, left (red line). It starts from zero, increases to reach the maximum, and drops to a zero at the end of the lean forward phase $\left(T_{\text {eLF }}\right)$. To determine the beginning of the transition, we first find the maximum angular velocity (v.LF) that is above a certain threshold, and then search backward to find the beginning of the slope $\left(\mathrm{T}_{\mathrm{bLF}}=\mathrm{T}_{\mathrm{bS} 2 \mathrm{ST}}\right)$. The maximum upper trunk angle, a.S2ST, is reached (Fig. 5, left) at the end of the lean forward phase. The time distance between the beginning of the transition and the end of the lean forward phase represents the duration of the lean forward phase, d.LF = $\mathrm{T}_{\text {eLF }}-\mathrm{T}_{\mathrm{bS} 2 \mathrm{ST}}$. The second phase of the sit-to-stand transition is characterized by a negative angular velocity as the subject moves into an upright position. The angular velocity reaches the minimum, v.LT, and then increases back to zero. The moment when it becomes positive is considered to be the end of the lift up phase and the end of the sit-to-stand transition. By time stamping this moment $\left(\mathrm{T}_{\mathrm{eS} 2 \mathrm{ST}}\right)$, we calculate the duration of the lift up phase, d.LT $=\mathrm{T}_{\mathrm{eS} 2 \mathrm{ST}}-\mathrm{T}_{\mathrm{eLF}}$, and the total duration of sit-to-stand transition, d.S2ST $=$ d.LF + d.LT.
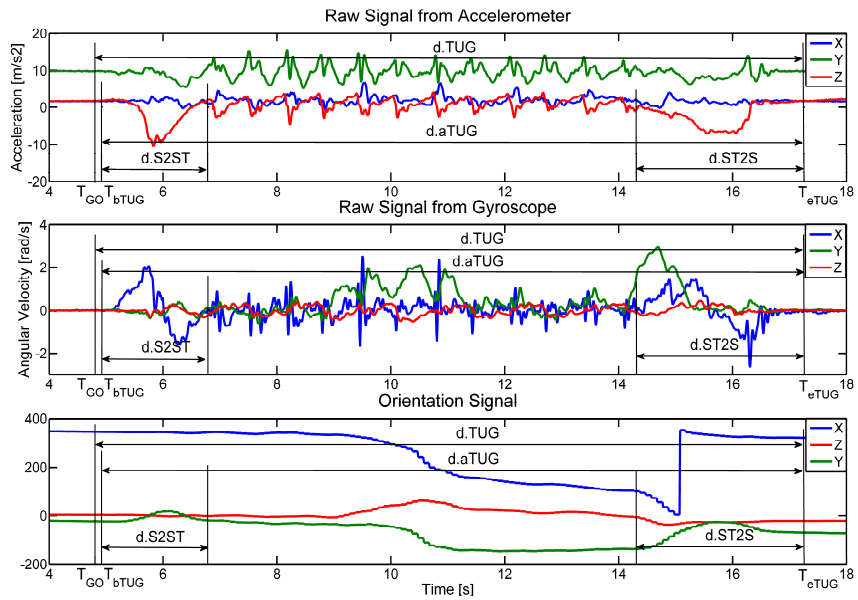

Fig. 4. Accelerometer, gyroscope and orientation signals (the $x, y, z$ components) recorded during the TUG test.

A stand-to-sit transition can also be divided into two separate phases, a prepare-to-sit (PS) and a sit-down (SD) phase. The angular velocity and the upper trunk angle profiles during the stand-to-sit transition are shown in Fig. 5, right. The 
angular velocity increases to the maximum and then drops back to zero as the subject leans forward in preparation to sit down. The moment when the angular velocity drops to zero marks the end of the prepare-to-sit phase and the beginning of the sitdown phase. By time stamping these characteristic points we can determine the duration of the preparation phase, d.PS = $\mathrm{T}_{\mathrm{ePS}}-\mathrm{T}_{\mathrm{bST2S}}$. In the sit-down phase, the angular velocity is negative as the subject's upper trunk moves back into the upright position. The moment it becomes positive marks the end of the sit-down phase $\left(\mathrm{T}_{\text {eST2S }}\right)$ and the entire stand-to-sit transition. By time stamping this moment we can calculate the duration of the sit-down phase, d.SD $=\mathrm{T}_{\mathrm{eST} 2 \mathrm{~S}}-\mathrm{T}_{\mathrm{ePS}}$, and the entire stand-to-sit transition (d.STS2 $=$ d.PS + d.SD).
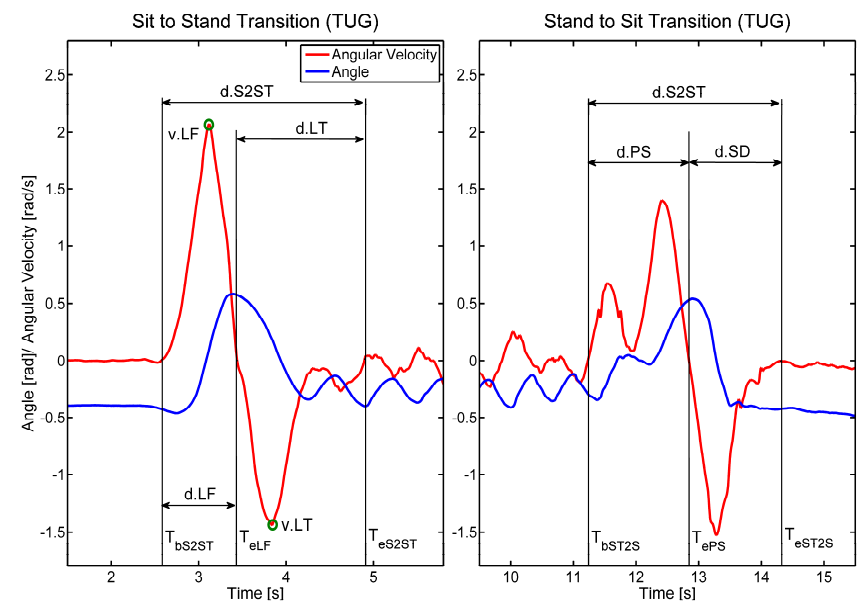

Fig. 5 Angular velocity and upper body trunk angle during the sit-to-stand (left) and stand-to-sit (right) transitions for TUG.

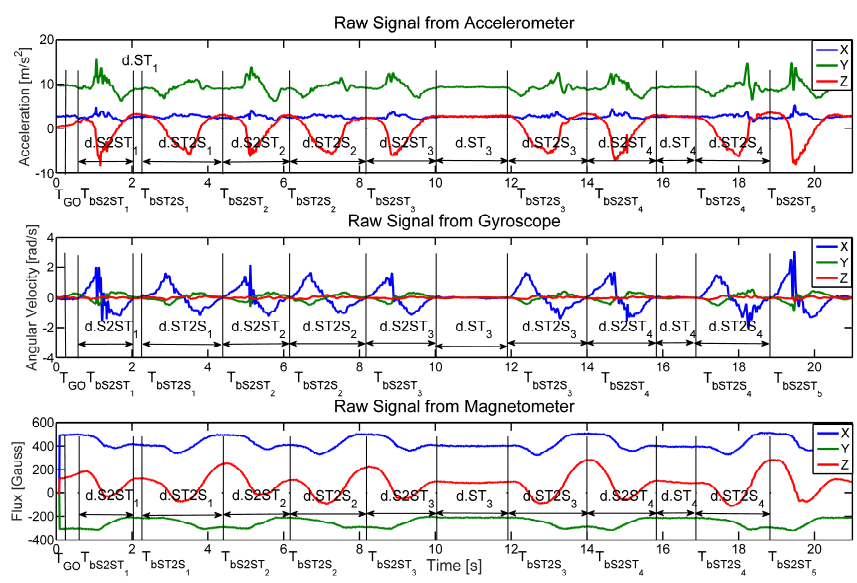

Fig. 6. Accelerometer, gyroscope and magnetometer signals (the $x, y, z$ components) recorded during first 4 cycles in the 30 SCS test.

Fig. 6 shows raw acceleration, angular velocity, and magnetic field signals captured for a portion of a 30SCS test with 4 cycles of chair stands. The plots are annotated with markers indicating relevant events for each cycle of a chair stand. The test starts when the command "Go" is issued. Similarly to the TUG test, we detect a standing up phase with its sub-phases (leaning forward and lifting up). A subject can spend some time in the standing position, described by a parameter $\mathrm{d} . \mathrm{ST}_{\mathrm{i}}$. The standing is followed by a stand-to-sit transition. In the sitting position, a subject may also stay for some time before a new cycle is started. The sitting is characterized by a parameter d.SIT . Please note that in healthy subjects the parameters d.ST $\mathrm{i}$ and d.SIT $\mathrm{S}_{\mathrm{i}}$ may be equal to zero, i.e., the subjects may move from one transition to the other without any delays. However, elderly people and people with balance and mobility impairments may require some time to prepare for the next cycle. Fig. 7 shows the profiles of the angular velocity for the sit-to-stand and the stand-to-sit transitions in 30SCS tests. To detect characteristic events, the starting and ending points of transitions, we use similar algorithms to those described for the TUG test.
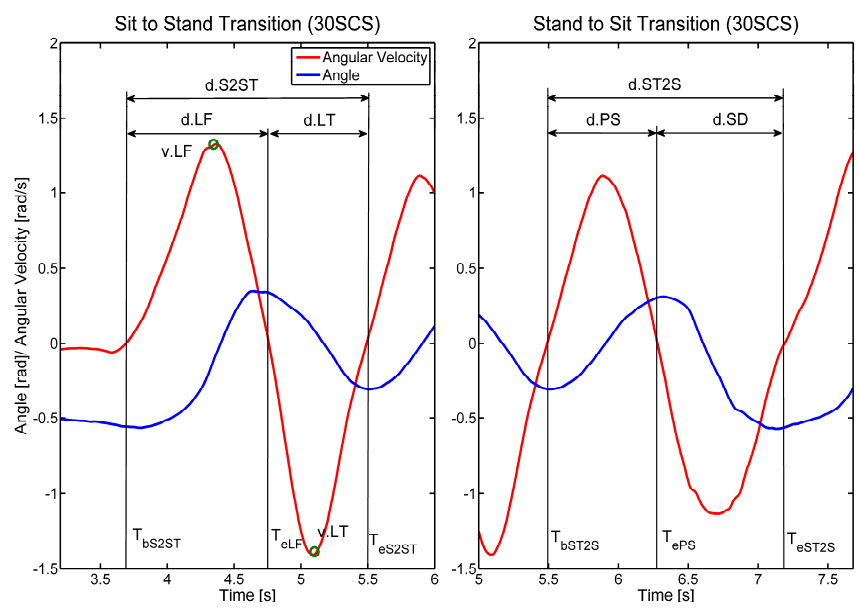

Fig. 7 Angular velocity and upper body trunk angle during the sit-to-stand (left) and stand-to-sit (right) transitions for 30SCS.

\section{Testing AND INITIAL RESUlts}

To verify the correctness of the Smart Button parameter extraction, we use a custom program that captures videos of the experiments and synchronizes them with other measurements and the cues. The videos are annotated manually to identify individual transitions and extract time parameters. These parameters are then compared to those extracted by the Smart Button. These tests proved that the Smart Button works accurately. In addition, the Smart Button feature extraction algorithms are verified in an Autonomous Tracking Optical Measurement (ATOM) Lab. The ATOM lab allows accurate tracking of reflective markers using 33 Vicon T40 series IR cameras. The Vicon system records the absolute position of each marker with millimeter precision every $20 \mathrm{~ms}$. The markers are placed on the Smart Button and the upper trunk of a test subject. The parameters characterizing posture transitions derived from the Vicon system are then compared to the ones reported by the Smart Button. We find that the Smart Button parameters match those derived from the Vicon system.

An initial testing of the Smart Button is performed on several healthy individuals. Each individual was asked to perform the TUG and 30SCS tests at their normal pace and each test is repeated three times. Table 4 shows the averaged results for the TUG tests that cover a diverse set of individuals.

Table 5 shows the result for a single 30SCS test. The subject completed 8 cycles in 30 seconds. For each stand chair cycle we give an extended summary, showing the duration of a cycle that includes (a) a transition for sitting to standing, (b) 
possibly standing still, (c) a transition from standing to sitting, and (d) possibly sitting before the next cycle is started. With this information we can observe whether a subject is taking more time to stand up toward the end of the 30SCS test. In addition, we can compare the times the subject spends in different phases, e.g., taking more time to stand up.

TABLE IV. THE TUG TEST REsUlts: AN EXAMPLE

\begin{tabular}{|l|c|c|c|c|}
\hline Subject & S1 & S2 & S3 & \multicolumn{1}{c|}{ S4 } \\
\hline d.TUG & 7.87 & 8.78 & 9.58 & 11.51 \\
\hline d.aTUG & 7.67 & 8.69 & 9.46 & 11.43 \\
\hline d.S2ST & 1.03 & 1.51 & 1.63 & 1.62 \\
\hline d.LF & 0.77 & 0.84 & 0.98 & 1.02 \\
\hline d.LT & 0.26 & 0.67 & 0.66 & 0.60 \\
\hline d.ST2S & 1.93 & 1.94 & 1.92 & 1.75 \\
\hline d.PS & 1.11 & 1.16 & 1.05 & 0.91 \\
\hline d.SD & 0.82 & 0.77 & 0.87 & 0.84 \\
\hline n.STEP & 9 & 12 & 13 & 12 \\
\hline
\end{tabular}

TABLE V. THE 30SCS TEST RESULTS: AN EXAMPLE

\begin{tabular}{|l|c|c|r|r|r|r|r|c|}
\hline Cycle (i) & $\mathbf{1}$ & $\mathbf{2}$ & $\mathbf{3}$ & $\mathbf{4}$ & $\mathbf{5}$ & $\mathbf{6}$ & $\mathbf{7}$ & $\mathbf{8}$ \\
\hline d.SCS $_{i}$ & 2.82 & 2.72 & 2.71 & 2.73 & 2.56 & 2.77 & 2.7 & 2.57 \\
\hline d.S2ST $_{i}$ & 0.89 & 1.14 & 0.8 & 1.26 & 1.15 & 0.89 & 1.16 & 1.08 \\
\hline d.LF $_{\mathrm{i}}$ & 0.55 & 0.39 & 0.49 & 0.55 & 0.49 & 0.52 & 0.49 & 0.43 \\
\hline d.LT $_{i}$ & 0.34 & 0.75 & 0.31 & 0.71 & 0.66 & 0.37 & 0.67 & 0.65 \\
\hline d.ST $_{i}$ & 0.44 & 0.01 & 0.43 & 0.01 & 0.01 & 0.38 & 0.01 & 0.01 \\
\hline d.ST2 $_{i}$ & 1.49 & 1.57 & 1.48 & 1.46 & 1.4 & 1.5 & 1.53 & 1.48 \\
\hline d.PS $_{i}$ & 0.74 & 0.75 & 0.72 & 0.62 & 0.61 & 0.67 & 0.66 & 0.61 \\
\hline d.SD $_{i}$ & 0.75 & 0.82 & 0.76 & 0.84 & 0.79 & 0.83 & 0.87 & 0.87 \\
\hline
\end{tabular}

\section{CONCLUSIONS}

Proliferation of tiny embedded computing platforms enables the design of smart devices for instrumentation and quantification of standard medical procedures. In this paper, we describe a new device in the form factor of a button that is used to assess mobility of elderly and people with Parkinson's disease. The device, called Smart Button, analyzes the upper body motion to fully quantify two tests that are frequently used to assess mobility of elderly: the Timed-Up-and-Go and 30Second Chair Stand tests. We describe a rich set of parameters that can be extracted from each test, offering health care professionals and researchers a new tool for analyzing longterm trends in the mobility of their patients. The accuracy of derived parameters is verified using a high-fidelity system.

Our plans are to expand the firmware to support other tests used to assess mobility and balance and to test the device in ambulatory conditions.

\section{REFERENCES}

[1] D. Podsiadlo and S. Richardson, "The timed 'Up \& Go': a test of basic functional mobility for frail elderly persons," J. Am. Geriatr. Soc., vol. 39, no. 2, pp. 142-148, Feb. 1991.

[2] C. J. Jones, R. E. Rikli, and W. C. Beam, "A 30-s chair-stand test as a measure of lower body strength in community-residing older adults," Res. Q. Exerc. Sport, vol. 70, no. 2, pp. 113-119, Jun. 1999.

[3] M. Csuka and D. J. McCarty, "Simple method for measurement of lower extremity muscle strength," Am. J. Med., vol. 78, no. 1, pp. 7781, Jan. 1985.

[4] B. Najafi, K. Aminian, F. Loew, Y. Blanc, and P. A. Robert, "Measurement of stand-sit and sit-stand transitions using a miniature gyroscope and its application in fall risk evaluation in the elderly," IEEE Trans. Biomed. Eng., vol. 49, no. 8, pp. 843-851, Aug. 2002.
[5] A. G. Society, G. Society, A. A. Of, and O. S. P. On Falls Prevention, "Guideline for the Prevention of Falls in Older Persons," J. Am. Geriatr. Soc., vol. 49, no. 5, pp. 664-672, May 2001.

[6] "Effects of Methylphenidate on Cognitive Function and Gait in... : Clinical Neuropharmacology," $L W W$. [Online]. Available: http://journals.lww.com/clinicalneuropharm/Fulltext/2006/01000/Effe cts of_Methylphenidate on Cognitive Function.5.aspx. [Accessed: 09-May-2015].

[7] R. Ben-Itzhak, N. Giladi, L. Gruendlinger, and J. M. Hausdorff, "Can Methylphenidate Reduce Fall Risk in Community-Living Older Adults? A Double-Blind, Single-Dose Cross-Over Study," J. Am. Geriatr. Soc., vol. 56, no. 4, pp. 695-700, Apr. 2008.

[8] S. R. Lord, S. M. Murray, K. Chapman, B. Munro, and A. Tiedemann, "Sit-to-Stand Performance Depends on Sensation, Speed, Balance, and Psychological Status in Addition to Strength in Older People," $J$. Gerontol. A. Biol. Sci. Med. Sci., vol. 57, no. 8, pp. M539-M543, Aug. 2002.

[9] S. L. Whitney, D. M. Wrisley, G. F. Marchetti, M. A. Gee, M. S. Redfern, and J. M. Furman, "Clinical Measurement of Sit-to-Stand Performance in People With Balance Disorders: Validity of Data for the Five-Times-Sit-to-Stand Test," Phys. Ther., vol. 85, no. 10, pp. 1034-1045, Oct. 2005.

[10] S. Buatois, D. Miljkovic, P. Manckoundia, R. Gueguen, P. Miget, G. Vançon, P. Perrin, and A. Benetos, "Five Times Sit to Stand Test Is a Predictor of Recurrent Falls in Healthy Community-Living Subjects Aged 65 and Older," J. Am. Geriatr. Soc., vol. 56, no. 8, pp. 15751577, Aug. 2008.

[11] A. Weiss, T. Herman, M. Plotnik, M. Brozgol, I. Maidan, N. Giladi, T. Gurevich, and J. M. Hausdorff, "Can an accelerometer enhance the utility of the Timed Up \& Go Test when evaluating patients with Parkinson's disease?," Med. Eng. Phys., vol. 32, no. 2, pp. 119-125, Mar. 2010

[12] C. Zampieri, A. Salarian, P. Carlson-Kuhta, K. Aminian, J. G. Nutt, and F. B. Horak, "The instrumented timed up and go test: potential outcome measure for disease modifying therapies in Parkinson's disease," J. Neurol. Neurosurg. Psychiatry, vol. 81, no. 2, pp. 171176, Feb. 2010.

[13] E. P. Doheny, C. W. Fan, T. Foran, B. R. Greene, C. Cunningham, and R. A. Kenny, "An instrumented sit-to-stand test used to examine differences between older fallers and non-fallers," in 2011 Annual International Conference of the IEEE Engineering in Medicine and Biology Society, EMBC, 2011, pp. 3063-3066.

[14] N. Millor, P. Lecumberri, M. Gómez, A. Martínez-Ramírez, and M. Izquierdo, "An evaluation of the 30-s chair stand test in older adults: frailty detection based on kinematic parameters from a single inertial unit," J. NeuroEngineering Rehabil., vol. 10, no. 1, p. 86, Aug. 2013.

[15] M. Marschollek, G. Nemitz, M. Gietzelt, K. H. Wolf, H. M. zu Schwabedissen, and R. Haux, "Predicting in-patient falls in a geriatric clinic," Z. Für Gerontol. Geriatr., vol. 42, no. 4, pp. 317-322, Jun. 2009.

[16] M. Milosevic, E. Jovanov, and A. Milenkovic, "Quantifying TimedUp-and-Go test: A smartphone implementation," in 2013 IEEE International Conference on Body Sensor Networks (BSN), 2013, pp. $1-6$.

[17] CDC, "The Timed Up and Go (TUG) Test." [Online]. Available: http://www.cdc.gov/homeandrecreationalsafety/pdf/steadi2015.04/TUG_Test-a.pdf. [Accessed: 15-May-2015].

[18] CDC, "30 Second Chair Stand Test." [Online]. Available: http://www.cdc.gov/homeandrecreationalsafety/pdf/steadi-

2015.04/30 Second Chair_Stand Test-a.pdf. [Accessed: 15-May2015].

[19] "Android Sensor Fusion Tutorial - CodeProject." [Online]. Available: http://www.codeproject.com/Articles/729759/Android-Sensor-FusionTutorial. [Accessed: 15-May-2015].

[20] M. Milosevic, A. Milenkovic, and E. Jovanov, "mHealth @ UAH: Computing Infrastructure for Mobile Health and Wellness Monitoring," XRDS, vol. 20, no. 2, pp. 43-49, Dec. 2013. 